\title{
Research of Manufacturing Resource Sharing System Based on P2P
}

\author{
Yunxia Jiang, Shuqi Wang, Minli Zheng and Dongnan Sun \\ School of Mechanical and Power Engineering, Harbin University Science and \\ Technology, Harbin, China \\ hrbustjyx@163.com
}

\begin{abstract}
To realize the equal sharing and full use of manufacturing resource, aiming at the convergence characteristics and dynamic changing behaviors of the distributed and stochastic manufacturing resources, according to the thought of peer-to-peer $(P 2 P)$ and the Chord protocol, a distributed manufacturing resource sharing system model is proposed, making logic sub rings by the type of resources and the logic main rings by the super nodes. The relay of the resource rings and the convergence of different types of resources for application are completed by super nodes. The description template of the manufacturing resources and the Meta data model relating to the search keyword sets from the template are designed. The indexing process of the resources based on Meta data model and the hierarchical trust mechanism of the sharing system are elaborated; finally, an instance of the sharing system prototype is given.
\end{abstract}

Keywords: P2P; Chord; manufacturing resource sharing; Meta data

\section{Introduction}

China today has become the big manufacturing country in the world, standing at a new historical starting point. But the manufacturing resources and manufacturing capacity of our country have the problem of strong regional distribution and it's hard to provide fair and reasonable use chance for the distributive manufacturing resources users. As the global manufacturing industry transforms from the traditional production manufacture to the service-oriented manufacture, and as the modern advanced cloud computing technology and physical information fusion technology emerge, manufacturers tend to search for the full sharing and the reasonable allocation methods of the manufacturing resources. For this, orienting to networked manufacturing, each enterprise, as the resources bearing point, cooperates with each other at different levels, and shares the manufacturing application tasks, in order to realize the convergence of resources and the integration of manufacturing processes [1]. This loose collaboration, based on the thought of the Internet resources sharing, needs an open and new information model and the supporting of the resource search architecture.

Centralized resource sharing structure [2] has been more mature. It emphasizes the accurate retrieval and inference, and by building a multi-level intelligent discovery retrieval model, it can support the precise retrieval, fuzzy retrieval, semantic retrieval and intelligent reasoning, etc of manufacturing resources. But this model has also exposed some problems, such as risk concentration, poor fault redundancy ability, high centralized storage management cost, hard management of the sharing data consistency and the lack of the independent management power of the own resources of enterprises and so on.

In contrast, P2P network model [3] emphasizes the equal status of system structure nodes, and the resources storage utilization is flexible on network and with a strong extensibility, P2P is more suitable for the convergence model of manufacturing 
resources. Distributed P2P network structure is divided into two types. One is unstructured $\mathrm{P} 2 \mathrm{P}$ architecture [4], and its query requests broadcast to all neighbors by flooding until meeting the query or timeout. The query efficiency is low and with poor scalability, but it has good robust performance. Another is structured P2P network [5], and it uses the distributed hash table (DHT) query mechanism. The query efficiency is high and with excellent expansibility. In networked manufacturing, the manufacturing resources nodes hope to randomly access and exit, which requires the strong scalability of resources sharing network. Two layers of DHT overlay network structure of application-oriented convergence is presented in this paper, based on which, the framework model of manufacturing resource sharing system is built. The Chord protocol is adopted to implement resources localization, and the Meta data is used to realize the search for basic resources.

\section{The Manufacturing Resources Sharing Model}

The existence of manufacturing resources is distributed, cross-regional and randomness. The discovery and retrieval of the resource service is more considering the extensibility and equality. The enterprise owning resources requires the mastership of the resources, and hopes to access or withdraw from the sharing system independently. Originally cross-regional manufacturing resources randomly access the resource sharing network, and the bottom changes will not affect the application of the upper system [5]. The users hope to get needed resources optimally and quickly, and which region or which enterprise to provide the resources is not the main concern for resource demanders primarily. For this, this paper divides the manufacturing resource sharing system structure into four layers, and guarantees the independent update and undertaking of each layer, to complete the function of resource sharing commonly, as shown in figure 1. Based on the relationship of resources serving and be served, in the system the enterprises owning the resources are defined as the providers of the resources, and the users are defined as the consumers. The process of manufacturing resource sharing is: in the distributed and heterogeneous network environment, resource consumers and providers realize the session of the resource registration, search starting, positioning, and optimizing the allocation and information obtaining.

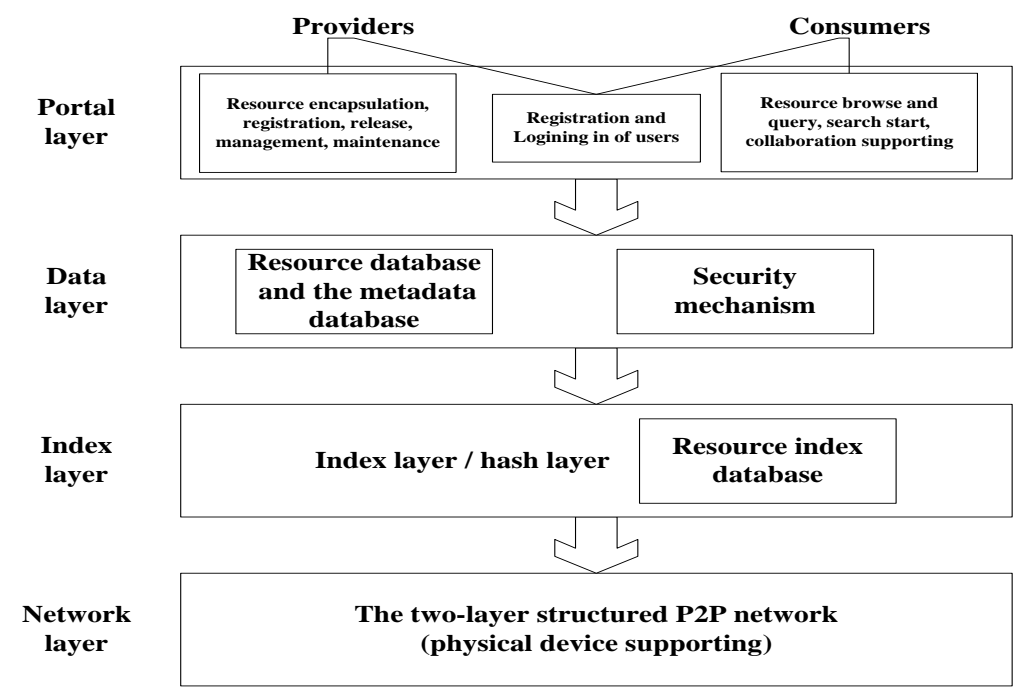

Figure 1. The Hierarchical Model of the Manufacturing Resource Sharing System 
Network layer is the network architecture of the system. The manufacturing resource nodes consist of the resource providers; here we call them Physical Nodes (PN). Each physical node interconnects through the network. Namely the physical node is added to the system with independent <IP, port> pair. For the loose organization of the distributed manufacturing resources in the system, a structured P2P network is used to construct the manufacturing resource network. The Chord agreement is selected as DHT resource search algorithm [6], and it is hierarchically extended to two kinds of Chord rings, forming the improved model of two layers of polycyclic Chord based on the resource classification.

Index layer realizes a simple and available distributed hash table based on the twolayer Chord ring, and each node saves a part of the hash table. In the resource sharing system, index layer is responsible for the storage of data and the access of the index keywords. Namely it can storage a $<$ key, value $>$ data to the mapping node and obtain the data from the mapping node. At the same time it storages the $<$ key, value $>$ data in the Mysql database to constitute the index database. This layer provides the upper with a very simple interface of get and put, and get or put function first maps the key to a specified node by using the two-layer Chord ring, after then, the access operation can be realized.

Data layer realizes the description and storage of the manufacturing resources, and the description of the resource Meta data. In the manufacturing resource sharing system, Meta data is used to describe the important attributes of manufacturing resources. It is the data about data, used to describe elements and the data sets and other information, mainly including coverage, quality, management mode, the owner of the data, the providing method of the data and main technical parameters and other relevant information. Meta data has been applied in many areas, and in the field of database it refers to the data about the data warehouse [7]. Each type of manufacturing resources uses different meta data to describe, which is called the meta data model of that kind of resources, such as gantry boring and milling machine, its meta data model refers to a set of attributes, Including the parameters of the processing scope, main spindle, precision and cutting tool constraints, and as the search keywords, this set can require the semantic search. It is conceivable that the Meta data model can improve the quality of the expression of manufacturing resources information, and regarding the Meta data model as the carrier, the search can reduce unnecessary network load and improve the search accuracy.

Portal layer is also a interaction interface layer of the resource sharing system, which provides the providers and consumers of the resources with the registration, enrollment and other management functions, and provides the resource providers with the interaction functions, such as resource encapsulation, release, maintenance, and management and so on, and provides the resource consumers with the resource query, search, and interactive interface of the collaborative supporting.

In all, the system provides users with the function of the share resources, search and release resources. According to the unified definition of some resource types, it defines global unified description model for each type of resources, namely the Meta data model, which is predefined in the system, and completed in the data layer. When each resource provider logins in, they access to the resource sharing system according to their own resource types. Data layer extracts attribute information from all shared resources and constructs index key phrases according to the sequence of their important degree, and then the key phrases are distributed to the specified nodes in the system. The data layer and index layer realize the storage of the key phrases and index information data, and after, the consumers search for the corresponding resources through the search interface. 


\section{Two-layer Polycyclic Improvement Structure of Chord}

The two-layer polycyclic Chord model, as an improved model of Chord based on resource classification, is a two-layer logical structure, as shown in Figure 2. The first layer is a like Chord structure that consists of Super Node (SN), called super ring, and it is the main ring in the model and mainly responsible communication between the subrings. The second layer is a resource ring that consists of logical nodes (LN) with the same type of manufacturing resources, also called resource subring. It is also a like Chord structure, and classified through resource type of the nodes. A physical node PN can have a variety of resources, namely the physical node can join different logical rings according to their own resources categories, to become $\mathrm{LN}$ of different logical ring.

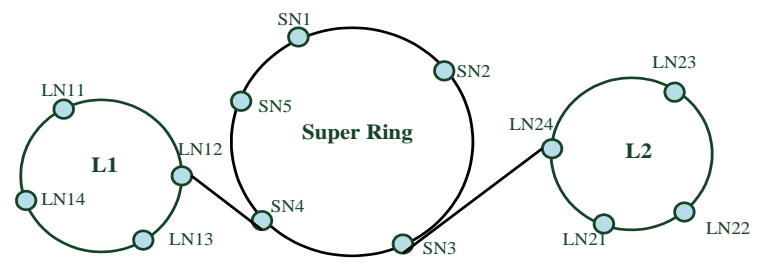

\section{Figure 2. Two-layer Polycyclic Chord Structure}

Each ring needs precursor nodes and subsequent nodes that are two data to bidirectionally connect to form ring, and it use finger table of the node to implement the routing search. Precursor and subsequent data structure are defined as a succ[] and pred[]. $\mathrm{SN}$ is the entry node of the resource ring, and also the nodes in the ring. It has at least two finger tables. One is used to maintain the super ring; another is used to maintain the resource ring. It forms a like Chord structure through the precursor and subsequent nodes and the finger tables. The clustering of manufacturing resources is realized according to the needs of the upper manufacturing tasks, and the same type resources are in a logical ring of the resources. LN of each resource ring maintains the subsequent node information of the entrance of the ring SN, namely the subsequent node list $\mathrm{S}$ _succ_list [] of the resource types, through which to find the entry node SN in the super ring. At the same time, due the entry node is dynamic, when the entry node changes, the network self-adaption reaches an agreement.

\subsection{The Join of Nodes}

In the dynamic network, due the two-layer ring is the logical ring, the nodes access can be divided into physical nodes access and logical nodes access. A new physical node may be added to the system at any time. Before the join, the access agent node $n$ 'that already exists in the system is needed [8]. Physical nodes access needs to treat two decisions: supposing physical node $\mathrm{n}$ joins, first determine whether $\mathrm{n}$ has the ability to be $\mathrm{SN}$. If yes, estimate the current SN number in the system, and if the SN number does not exceed thresholds, the $\mathrm{n}$ joins as SN. After, based on the types of resources in $\mathrm{n}, \mathrm{n}$ joins the corresponding resource ring as the logical nodes.

The join of logical nodes is dynamic, and physical nodes in running can add new resource type or delete a resource type, leading to the increasing or deleting of resource nodes or rings. But as SN, unless physical nodes quit or are replaced, it can not be changed. When LN accesses, if some resource type does not exist in the initial state of the system, the system needs whether to choose $\mathrm{SN}$ to create resource ring. If node joins the super ring to become $\mathrm{SN}$, it may replace other nodes to be the entrance of some resource ring. And the replaced SN automatically converts to the ordinary LN, and it is labeled for backup SN. The basic access process is: through the IP address of the access node obtain the identifier ID $=\mathrm{n}$ of the hash function calculation; through a proxy node $n$ 
', $\mathrm{n}$ find its own subsequent node ID $=\mathrm{m}$. Set the subsequent node $\operatorname{succ}[\mathrm{n}]=\mathrm{m}$, and inform the subsequent node to modify its precursor node $\operatorname{pred}[\mathrm{m}]=\mathrm{n}$. At last, the access node requests again $\mathrm{n}$ ' to find the entrance $\mathrm{SN}$ and configurates $\mathrm{r}_{-}$succ_list of resource. Set precursor node and update the finger table, as well as use precursors to update subsequent nodes.

\subsection{The Quit and Failure of Nodes}

Nodes can be free to join or quit, and its quit is also dynamic. If quits, it is required to send out the quit initiatively, and the other nodes immediately update their status table to ensure the dynamic adaptability of the overlay network. When LN node leaves the overlay network, it needs to inform other nodes in area initiatively to replicate its resource index information, and other nodes will immediately update the hash table for their own maintenance to ensure the efficiency of message routing [9]. If the SN quits, in addition executing the same exit steps, the backup SN also needs to start.

The quit or failure of physical nodes leads to the failure of logical node, and at the same time the cancellation of some resources in the physical node will lead to the failure of LN. Based on the principle of Cord, when LN node fails, its precursor nodes realized the link connection again by maintaining a certain number of subsequent lists. When subsequent node fails, it choose subsequent successor from the list to implement the link connection. If SN node fails, backup nodes competitively select new SN, and refresh routing information itself.

\section{Search based on Metadata}

\subsection{Predefined Metadata Model}

In the manufacturing resource sharing system, each type of resource should have the unified property description, here to construct a description template of resource class. At the same time, in order to consistent semantic of query keywords, the data layer defines a metadata model for each type of resource, the metadata is used to describe the important properties of resources, to serve as an index model for each type of resource search, so that the resources can acquire the global unified management[10].

Resource description template has a clear definition of concept for the manufacturing resources in reality, can be formally described as PS::=<Name, BP, AP, TP, RS $>$, and Name is on behalf of the name of the manufacturing resources, to define and describe the concept identifiers of the manufacturing resources. BP reflects the static attribute information collection of manufacturing resources that actual exist, such as subordinate enterprises, contact information, etc.; AP reflects the dynamic attribute information set of manufacturing resources, such as the current state, use frequency, etc.; TP corresponds the technical parameter information attribute set of manufacturing resources, such as the processing range of milling machine, precision ,etc.; RS is the constraint of manufacturing resources, such as the mutual constraints between machine tools and cutting tools.

The metadata model extracts from resource description template, and predefines in order to meet the manufacturing task and the resource consumer demand, formal description is UI::=<Classn, KBI, KTP, KRS $>$. Classn is the identification of manufacturing resource class and resource name, Classn can be used as search keywords of class, but also search keywords of resource instances of a class; KBI is the search collection of key information of resource; KTP represents the search collection of key technical parameters; KRS represents key associated parameters collection of the resources.

The metadata model is a set of attributes, so the metadata model corresponds a plurality of keyword indexing. Resource providers login system, and issue each resource 
instances under each type of resources .The data layer extracts the resources attribute information set according to the corresponding metadata model, to composite attributeattribute value pair collection, respectively as the hash key to go on the hash computation, to generate multiple < key, value > index data collection, if the attribute value is empty, not its processing.

In the resource sharing system, the data layer can be put and get $\langle$ key, value $>$, in order to launch resource search on a network. According to a property of resource, the key words indexing <key, value> is generated, can be described as : (1)key: a attributeattribute value pair that is hash value in the metadata model; (2)value: It refers to Resource identifiers and resource location information, the resource identifier is the hash value of resource content, and resource location information can use the URL to represent. Because of the existence of the resource identifier, even the definite names of the resources being different, the resources characteristics are basically identical, this makes the system adapt to the search of resources service ability, in resource characteristics as keywords, to meet the mission requirement that don't care about enterprise and region that resources belong to, only hope to obtain the optimal configuration of resource service.

\subsection{Resources Search of the System}

Resource search process of two layers Chord ring is search process of the cooperation of super node and resource node, on the basis of the different location of resource consumers, can be specifically divided into three kinds of circumstances:

1. When a node of super ring applies for search resources, firstly, according to the Chord routing protocol, the resource ring is found, where the resource types in, and it means that the entrance node of resource ring of (Super Node) is found, then by the entrance of super nodes, the resources search has been to in the resources ring, and the results have been returned to the node of the initiate request.

2. When the node on resources ring searches resources, first to see whether the locality store the index of the resource type, if there is, then to search in this resource ring; if you can't find, then to find the super node of the entrance of this resources ring. In the super ring, the entrance SN of this resource type can be acquired. Finally by the entrance SN in their own resources ring, the resource search has been gone on and the result have been returned.

3. When the node outside the ring applies for the search resources, a common interface node is provided by the distributed storage network. Interface node first finds the super nodes of entrance of resource type storage, after then the super node goes on the resource search.

The search keywords that resource consumers enter, form the multi groups < attribute query keyword >, and the data layer makes use of the consistent hashing function to calculate each group < attribute - query keywords > and get the set of key [], then through the get function of the data layer, and send request to each key in the collection. The node that obtain receipts of a request search from the local keyword index set stored in hash table, and return the resource location data of the matching key to the client. Until the results of all items in key [] return, the resource consumers finally get the results collection URL (resource). The data layer then performs connection operation for these collections to return the result to the user layer. For example, the user query the milling machine of "processing method = end milling, milling thickness $=3 \mathrm{um}$ ", for the query of each keyword indexes, the lower searches and returns two result set that respectively are [resource1, resource2, resource3 resource4] and [resource2 resource4, resource6, resource7], to take the intersection to get [resource 2, resource 4]. And then parameters, 
such as processing time, processing cost, etc., have been selected to go on the further optimization configuration, to obtain the optimal query result. The data layer for resource search provides the upper with the interface of p_Resource () and s_ch (R, set). P_Resource () bases on the metadata model to extract the properties from resource, to build the keyword index, and to store them into the network. S_ch (R, set) obtains a set of query keywords of pending query resources, and structure search keyword index to respectively seek. The result that is in-connected or summed is returned to the user layer.

When looking for, whether the local has SN has a certain effect on the search process. The request between the two resource rings is forwarded through the super node SN. For example, the resource sub rings L1, wanting to send a lookup message to resource sub ring L2, sends outward request, to use resources access SNR1 to forward in L1 ring, SNR1 on the super ring indexes the entrance SNR2 of L2, then by SNR2 the requests are forwarded to the L2 ring.

\section{The Network Trust Mechanism}

In the environment of P2P network, the equality of communication nodes is a common characteristic of many application systems. And it is requested that all nodes must be unconditional to obey unified organization principle and communication standards [11]. The network trust mechanism has to inhibit privilege, and to guarantee the equality of all nodes. At the same time, in the resources application system, the security and availability of resource directly affect the quality of service. Common security solution plan in the peer-to-peer network environment is a trust mechanism to build a resource. The basic method is to give the resource provider an evaluation by resource applicants after the transaction is completed, the results of multiple evaluations produce a trust. After the later query, the other resources applicants use the trust as reference, to choose more reliable resource provider.

This article uses the hierarchical trust mechanism. Within the logical ring, LN is the node that has measure weights up to standard such as credibility, online time and storage capacity etc., SN will regularly monitor transaction record parameters of LN, LN who's number of trust below a certain threshold is deleted automatically, to ensure that LN in each logical resource ring area with high credibility and security.

Security between LNS belongs to the safety of resources ring. The system adopts network reputation value (set as ns) to measure; Network reputation value is the weighted average of its own evaluation value and evaluation value of the other nodes which is contained in routing table. The value of $\mathrm{ns}$ is the basis to select resource providers. The security between $\mathrm{SN}$ is the safety between resource rings, in the same way, network reputation value ns is the first factor for choosing SN. Generally setting the connecting load rate of $\mathrm{SN}$ is the percentage between the number of LN and capacity of the SN. When connecting load rate is too high, then the system starts the increasing SN algorithm, new SN node's network reputation evaluation value must exceed the threshold. On this basis, the other elements are considered such as the resource categories, online time and capacity value etc. When the new LN access, it will start the evaluation of network credit value of access SN in the domain. If it can not meet the threshold, then the increasing SN algorithm will be started to ensure the security of SN. This method of hierarchical trust ensures the safe and reliable of resource trading process.

\section{Prototype Instance}

On the basis of the above research, milling CNC machine tools are selected, to develop the sharing prototype system of distributed machining equipment. To use the Openstack cloud computing platform to simulate the network topology of distributed manufacturing resources, resource gathering network of two layers resemble Cord 
structure has been built on it. Lathe-type resources and milling-type machine resources construct two logical resource rings, defined as L1 and L2, respectively, each virtual machine sets as a physical nodes PN, at least having the information of a machining equipment resources on each $\mathrm{PN}$, in order to simulate the LN on the corresponding resources ring, and to implement distributed resource pool. The super node ring is constructed by selecting the node in the two resource rings as the super node SN.

The portal system of prototype is shown in Figure 3, according to the resource types, different resource publishing interfaces are shown. In order to reduce the complexity of search, the system provides two types of search: according to the resource type, the feature search interface is expanded, as shown in Figure 3 (d); according to the requirements of mission, the task release interface is expanded, as shown in Figure 3(c). According to the matching rules, input information of the features search interface match up with KTP of the resource metadata to establish search keywords set. The fuzzy search has been used to traverse all the resources of meeting keywords set, combined with the optimization algorithm, to determine the final search information. Because the mechanical processing, machine tool, cutting tool and workpiece constitute a system, and associate with each other, the matching rules base can be establish through finding the corresponding relation of the characteristics of the workpiece processing and the corresponding relation of machine parameters.

Input information of the task release interface directly correspond with the metadata model UI, from the name to basic information, and to the technological parameters, keyword set is built in turn. By the attributes of name and basic information, the resource direct search can be realized, then the technical parameters as the auxiliary reference.

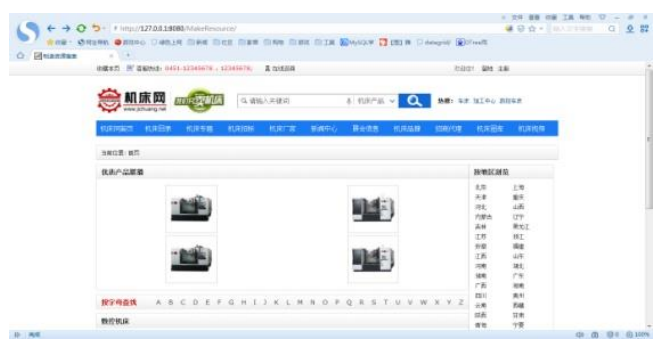

(a) Access to resources page

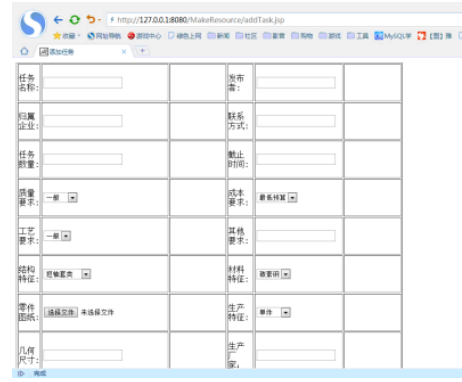

(c) Task release interf

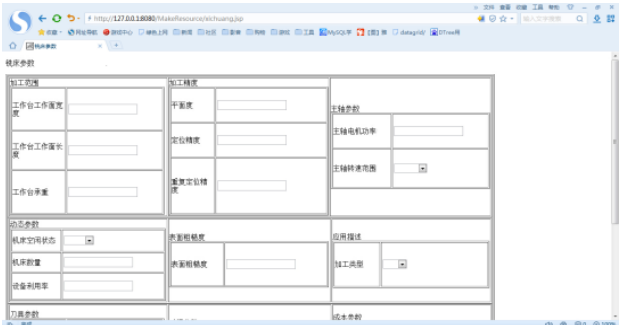

(b) Lathe publishing interface

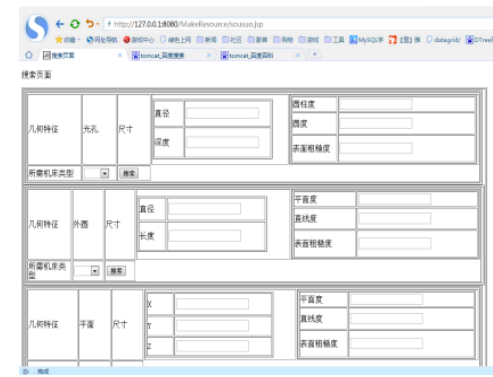

(d) Feature search interface

Figure 3. The Portal Page of the Manufacturing Resource Sharing System

\section{Conclusion}

A sharing platform of distributed manufacturing resources is proposed, based on two layers resemble Chord structure, and the sharing model realizes, to a certain extent, the independent and loose coupling between the underlying resources and upper application. This paper describes the two layer convergence network structure of the distributed resource nodes, designs and achieves the resource search mechanism and network trust mechanism based on metadata model. And on 
the basis of Sharing model, the sharing system of the turning and milling machine tool has been built, the keywords set of element model and the corresponding search and publishing interface is given, but the resource sharing incentive mechanism and the superiority of two layers of resembling Chord structure network need to be studied further, in the future, as the cloud manufacturing having been proposed and the cloud computing technology is gradually perfecting, how to integrate the sharing model into cloud manufacturing, to improve the efficiency of resources convergence need to be in-depth studied.

\section{References}

[1] L. M. Camarinha-Matos, H. Afsarmanesh and Gal-Eano, "Collaborative networked organizationsconcept and practice in manufacturing enterprise", Computers \& Industrial Engineering, vol. 57, no. 1, (2009), pp. 46-60.

[2] C. Ming, Z. Kai and L. Shanting, "Intelligent Discovery System of Manufacturing Resources oriented the Internet", Journal of computer aided design and graphics, vol. 21, no. 12, (2009), pp. 1820-1831.

[3] T. Dongming, L. Xianliang and Y. Lei, "Unstructured P2P search mechanism based on ant colony optimization", Advances in Information Sciences and Service Sciences, vol. 4, no. 11, (2012), pp. 257 263.

[4] E. Meshkova, Riihijvi and M. Petrova, "A survey on resource discovery mechanisms, peer-to-peer and service discovery frameworks", Computer Networks, vol. 52, no. 11, (2008), pp. 2097-2128.

[5] L. Shijun, Q. Benke, W. Lei and M. Xiangxu, "Research on gathered framework of self-organizing cloud manufacturing resources and searching method of multidimensional attribute range", Journal of computer aided design and graphics, vol. 24, no. 3, (2012), pp. 299-307.

[6] Z. Jingtao and W. Haicheng, "Enterprise information integration framework oriented equivalence of semantic grid service", Computer integrated manufacturing system, vol. 16, no. 12, (2010), pp. 32-39.

[7] D. G. Zhang, Y. X. Hu and D. Wang, "A new algorithm of service discovery based on DHT for mobile application", Journal of Network, vol. 6, no. 10, (2011), pp. 1466-1-474.

[8] B. Q. Wang, "Research and improvement of Chord routing algorithm", Computer Engineering and Applications, vol. 46, no. 14, (2010), pp. 112-114.

[9] J. W. Yin, W. Y. Zhang and Y. Li, "A peer-to-peer based multi-agent framework for decentralized grid workflow management in collaborative design", Int J Adv Manuf Technol, vol. 41, (2009), pp. 407-420.

[10] L. Gao, Y. M. Zhang and X. Wu, "Improvement of Chord search algorithm research in P2P network", Application Research of Computers, vol. 31, no. 2, (2014), pp. 158-521.

[11] T. Zhenhua, W. Xingwei and Z. Zhiliang, "The P2P distributed trust evaluation model based on multidimensional history vector”, Journal of computer, vol. 9, no. 33, (2010), pp. 1725-1735. 
International Journal of Grid Distribution Computing Vol.7, No.6 (2014) 